Arq. Bras. Med. Vet. Zootec., v.68, n.5, p.1207-1211, 2016

\title{
Provável extrusão de núcleo pulposo aguda e não compressiva em um cão: relato de caso
}

[Probable acute non-compressive nucleus pulposus extrusion in a dog: case report]

\author{
G.G. Franco ${ }^{1}$, E.G.M.D. Siqueira1 ${ }^{1}$ J.A.L.D. Souza ${ }^{1}$,L.O.D.C. Prado ${ }^{1}$, C.V.S. Brandão1, \\ S.C. Rahal ${ }^{1}$, M.J. Mamprim ${ }^{1}$, J.G. Quitzan ${ }^{1}$, B.W. Minto ${ }^{2}$ \\ ${ }^{1}$ Universidade Estadual Paulista - Unesp - Botucatu, SP \\ ${ }^{2}$ Universidade Estadual Paulista - Unesp - Jaboticabal, SP
}

\begin{abstract}
RESUMO
A extrusão discal aguda e não compressiva é caracterizada pela extrusão de caráter agudo/hiperagudo e não compressivo do núcleo pulposo de um disco intervertebral não degenerado. Pode ser chamada de hérnia de disco de baixo volume e alta velocidade ou explosões discais e geralmente está associado a exercícios intensos ou episódios traumáticos. O núcleo pulposo é fortemente forçado através de uma pequena fissura no ânulo fibroso dorsal, provocando uma contusão espinhal. Este relato tem como objetivo apresentar um caso de provável extrusão aguda de núcleo pulposo não compressiva. Foi atendido um cão macho, três anos e seis meses de idade, maltês, pesando $4,1 \mathrm{~kg}$. Como queixa principal, o proprietário relatou dificuldade locomotora e dor à manipulação há um dia, sem histórico de trauma. Foi constatada paraparesia não ambulatória de início agudo com ausência de propriocepção e dor superficial em membros pélvicos e dor à palpação epaxial da coluna toracolombar. A ressonância magnética (RM) evidenciou extensa área de hipersinal em segmento toracolombar da medula espinhal, sem sinais de compressão medular e de atenuação da intensidade do núcleo pulposo do disco intervertebral L1-L2. Foi feito diagnóstico presuntivo de mielopatia focal não compressiva com edema medular de todo segmento toracolombar, característico de uma extrusão aguda de núcleo pulposo não compressiva. Foi prescrito tratamento com anti-inflamatório esteroidal, analgésico, repouso absoluto e protocolo de reabilitação com acupuntura e fisioterapia. Após sete dias de tratamento, o animal recuperou a sensibilidade dolorosa superficial em membros pélvicos e evoluiu para paraparesia ambulatória. Os resultados deste relato sugerem que a RM pode ser útil para fazer um diagnóstico presuntivo em cães com histórico e sinais clínicos compatíveis. Além disso, o tratamento conservativo em extrusões discais não compressivas é preconizado e o paciente pode apresentar boa recuperação.
\end{abstract}

Palavras-chave: extrusão, núcleo pulposo, doença do disco intervertebral, lesão da medula espinhal

\begin{abstract}
Acute and non-compressive disc extrusion is characterized by the acute character of extrusion of the nucleus pulposus without real compression of the spine. It has been called low-volume and high speed disc herniation or disc explosions, and usually is associated with an intense exercise or traumatic episode. This report aims to present a case of an acute extrusion of nucleus pulposus with no compression of the spinal cord. A 3.5 year-old male dog of the Maltes breed, weighing $4.1 \mathrm{~kg}$ was presented at the Veterinary Hospital with locomotion disorders and pain during manipulation with no history of trauma. At the physical and neurological examination, non-ambulatory paraparesis of acute onset with absence of proprioception and superficial pain in hind limbs was found, as well as pain on palpation of epaxial thoracolumbar spine. Magnetic resonance imaging (MRI) showed extensive hyper intense area in the thoracolumbar spinal cord, with no signs of spinal cord compression, and decreased intensity of the nucleus pulposus of the L1-L2 intervertebral disc. Additionally, a spinal cord edema in all thoracolumbar segments was seen that is characteristic of an acute extrusion of non-compressive nucleus pulposus. A presumptive diagnosis of non-compressive myelopathy was assumed. The dog was prescribed steroidal anti-inflammatory, analgesic, absolute rest and rehabilitation
\end{abstract}

Recebido em 28 de setembro de 2015

Aceito em 1 de março de 2016

E-mail: guilherme.franco.vet@gmail.com 
protocol, including acupuncture and physiotherapy. The patient recovered superficial pain in the pelvic limbs and evolved into ambulatory paraparesis after seven days. The results of this report suggested that MRI can be useful for making a presumptive diagnosis in dogs with a history of compatible clinical signs. Moreover, the conservative treatment in non-compressive disc extrusions can be feasible.

Keywords: extrusion, nucleus pulposus, intervertebral disc disease, spinal cord injury

\section{INTRODUÇÃO}

Classicamente, as hérnias de disco podem ser classificadas em dois tipos principais, Hansen tipo I e tipo II. Posteriormente, foi proposta uma terceira classificação para as hérnias de disco agudas, porém não compressivas.

A extrusão discal aguda e não compressiva foi primariamente descrita por Griffiths (1970) e é caracterizada pela extrusão de caráter agudo ou hiperagudo e não compressivo do núcleo pulposo de um disco intervertebral não degenerado, diferentemente do tipo I, em que há degeneração condroide prévia (Chow et al., 2012). Pode ser chamada de hérnia de disco de baixo volume e alta velocidade, explosões discais, extrusão de disco traumática, explosão dorsolateral do disco intervertebral, doença do disco intervertebral Hansen tipo III (Griffiths, 1970; Chow et al., 2012; Henke et al., 2013). O termo extrusão do núcleo pulposo aguda e não compressiva (Enpanc) é usado aqui, pois descreve as principais características da doença e ajuda a diferenciá-la do tipo mais comum de extrusão de disco que ocorre após a degeneração do disco intervertebral (doença do disco intervertebral Hansen tipo I) e que normalmente resulta em contusão da medula espinhal e compressão.

$\mathrm{Na}$ Enpanc, o núcleo pulposo é fortemente forçado através de uma pequena fissura no ânulo fibroso dorsal, provocando uma contusão espinhal e, embora possa haver compressão transitória muito suave devido ao edema e/ou à hemorragia focal, não há etiologia compressiva contínua. O núcleo pulposo não degenerado dissipa-se rapidamente no espaço peridural (Risio et al., 2009).

Cães com Enpanc geralmente apresentam sinais de mielopatia aguda, frequentemente assimétrica e não progressiva após as primeiras 24 horas. A apresentação clínica da doença, os sinais clínicos, os achados na análise do líquido cefalorraquidiano e os achados da mielografia são muito semelhantes aos descritos para mielopatia isquêmica. A ressonância magnética (RM) fornece imagens que, associadas à experiência na interpretação, podem ajudar a diferenciar essas duas doenças (Risio et al., 2009).

O diagnóstico definitivo de Enpanc só é possível pelo exame post mortem por visualização macroscópica e exame histológico do núcleo pulposo gelatinoso extrudido para dentro do canal vertebral, o anel fibroso rompido, e da medula espinhal contundida.

$\mathrm{O}$ maior acesso à $\mathrm{RM}$ resultou em um aumento no número de animais em que o diagnóstico de Enpanc é feito in vivo, no entanto ainda existem poucos dados disponíveis sobre os achados da imagem, bem como o seu real papel prognóstico até o presente momento (Henke et al., 2013).

Este relato tem como objetivo descrever os achados do exame neurológico e da ressonância magnética, bem como sua evolução clínica em um caso de provável extrusão aguda e não compressiva (Enpanc) de núcleo pulposo em um cão.

\section{CASUÍSTICA}

Um cão macho, com três anos e seis meses de idade, maltês, pesando $4,1 \mathrm{~kg}$, foi atendido no Serviço de Cirurgia de Pequenos Animais da Faculdade de Medicina Veterinária e Zootecnia (Unesp - Botucatu). Como queixa principal, o proprietário relatou dificuldade locomotora e dor à manipulação há um dia, sem histórico de trauma. Ao exame físico e neurológico, foi constatado estado mental e função dos nervos cranianos normais. $\mathrm{Na}$ avaliação da deambulação, apresentou paraparesia não ambulatória de início agudo com ausência de propriocepção em membros pélvicos, dor superficial ausente e dor profunda presente em membros pélvicos e dor à palpação epaxial de coluna toracolombar. Outras alterações neurológicas ou ortopédicas não foram encontradas, bem como nos exames sanguíneos 
de rotina (hemograma e dosagens séricas de ureia, creatinina, alanina transaminase, proteína total e albumina).

A localização neuroanatômica da lesão foi no segmento toracolombar (T3-L3). Com os achados da anamnese, do exame físico e do exame neurológico, suspeitou-se como primeiro diagnóstico diferencial a extrusão discal Hansen tipo I.

O exame radiográfico mostrou discreta opacificação em espaço intervertebral de L1-L2. Os demais espaços e forames intervertebrais estavam preservados radiograficamente.

A ressonância magnética (Vet-MR Grande, campo 0,25 Tesla, Esaote) evidenciou extensa área de hipersinal em segmento toracolombar de medula espinhal, sem sinais de compressão medular e de atenuação da intensidade do núcleo pulposo do disco intervertebral L1-L2, visualizada nos cortes sagitais ponderados em T2 "Fast Spin Echo" (FSE) e "Short TI Inversion Recovery” (STIR) (Fig. 1). Foi feito diagnóstico presuntivo de mielopatia focal não compressiva com edema medular em todo o segmento toracolombar, característico de uma extrusão aguda de núcleo pulposo não compressiva. A análise do líquido cefalorraquidiano, que poderia ter ajudado a descartar doença inflamatória, foi recusada pelo proprietário.

Foi prescrito tratamento com prednisona $(0,5 \mathrm{mg} / \mathrm{kg}$ a cada 12 horas por três dias e depois $0,5 \mathrm{mg} / \mathrm{kg}$ a cada 24 horas por mais três dias), cloridrato de tramadol $(4 \mathrm{mg} / \mathrm{kg}$ a cada oito horas por cinco dias) e omeprazol $(1 \mathrm{mg} / \mathrm{kg}$ a cada 24 horas por seis dias). Além disso, foi recomendado repouso absoluto e protocolo de reabilitação com acupuntura e fisioterapia.

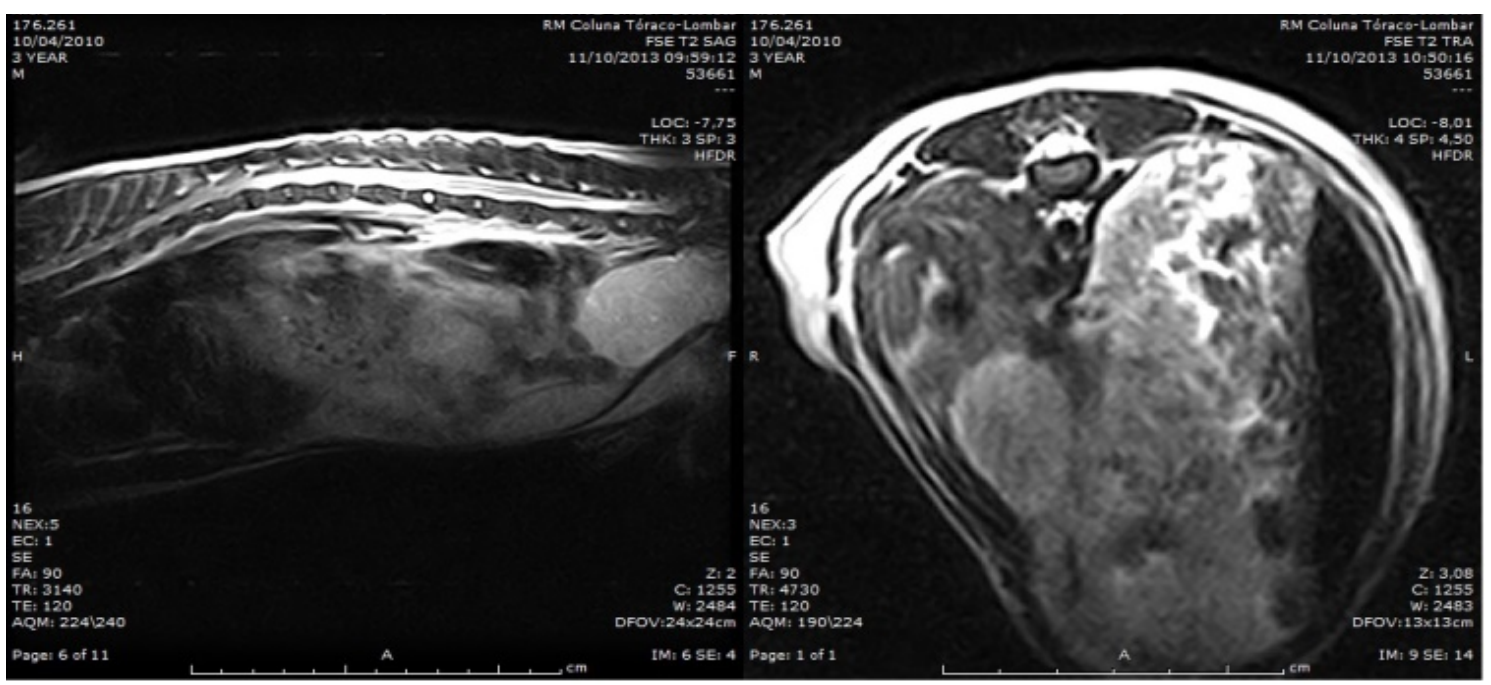

Figura 1: Ressonância magnética do segmento toracolombar da coluna vertebral nos planos sagital e transversal do espaço intervertebral L1-L2, mostrando uma extensa área de hipersinal em segmento toracolombar de medula espinhal, sem sinais de compressão medular (Vet-MR Grande, campo 0,25 Tesla, Esaote).

Após sete dias de tratamento, o animal recuperou a sensibilidade dolorosa superficial em membros pélvicos, e a paresia dos membros pélvicos diminuiu de intensidade, permitindo a deambulação, mas a ataxia proprioceptiva se manteve. Após o término do tratamento medicamentoso e com a realização do protocolo de reabilitação por meio de fisioterapia e acupuntura, o paciente apresentou melhora gradativa do quadro. Com 30 dias de evolução, houve melhora acentuada do quadro neurológico, sendo observada somente uma ataxia proprioceptiva discreta residual de membros pélvicos.

\section{DISCUSSÃO}

A extrusão de núcleo pulposo aguda e não compressiva presuntiva em cães tem sido descrita em diversos relatos de casos, a partir dos quais informações sobre a etiopatogenia, as características clínicas e os resultados de RM podem ser extrapolados. Um estudo com 42 
casos de suspeita de hérnia de disco aguda e não compressiva em cães demonstrou um bom resultado em 28 cães e um resultado ruim em 14 cães, em que sete apresentaram um aumento da gravidade dos sinais neurológicos associado ao aumento da área e do comprimento da lesão intramedular em imagens ponderadas em T2 de ressonância magnética (Risio et al., 2009). Neste relato de caso, em que os sinais neurológicos iniciais eram graves e a imagem da lesão medular na ressonância era extensa, o manejo conservativo apresentou bons resultados, embora uma ataxia residual tenha permanecido por três meses. Houve total retorno à função dentro de quatro semanas. Apesar dagravidade dos sinais neurológicos e daextensão da hiperintensidade intramedular em imagens de RM sagital e transversal ponderada em T2 estarem associadas ao resultado (Risio et al., 2009), a boa evolução deste caso pode ter sido o resultado de um trauma inicial menos grave da medula espinhal e o não desenvolvimento de lesões secundárias como mielomalácia e degeneração e morte neuronal irreversíveis.

A localização neuroanatômica clínica das lesões desse mesmo estudo foi nos segmentos da medula espinhal C1-C5 ( $\mathrm{n}=6)$, C6-T2 (6), T3L3 (28) e L4-S3 (2), sendo, portanto, o segmento toracolombar o mais frequente. $\mathrm{O}$ paciente aqui relatado apresentou a lesão localizada no segmento torcacolombar T3-L3.

A Enpanc em cães está frequentemente associada com exercício físico intenso (Mckee et al., 2010) ou com lesão traumática (Henke et al., 2013). Têm sido descritos casos em cães nos quais o núcleo pulposo dilacera a dura-máter ou se aloja no interior do parênquima medular (Yarrow e Jeffery, 2000). No caso descrito aqui, nenhum evento traumático ou atividade extenuante foi relatado, mas essa possibilidade não pode ser completamente excluída.

Em um estudo que avaliou os achados de imagem da RM em 31 casos de extrusão de disco intervertebral traumática, $71 \%$ dos casos não apresentavam lesões compressivas da medula, e os $29 \%$ que apresentaram lesão compressiva eram significantemente mais velhos e condrodistróficos (Henke et al., 2013). Corroborando os achados deste estudo retrospectivo, o paciente deste relato possui as características de idade e raça que aumentam a predisposição a uma lesão não compressiva em uma extrusão de disco aguda, principalmente se uma condição traumática ou exercício físico intenso estiver presente.

Como o diagnóstico definitivo de Enpanc só é possível pelo exame post mortem por visualização macroscópica e exame histológico do núcleo pulposo gelatinoso extrudido para dentro do canal vertebral, o anel fibroso rompido, e da medula espinhal contundida (Chang et al., 2007), é provável que atualmente essa doença seja subdiagnosticada pelo acesso limitado aos métodos de imagem avançados. Provavelmente é menos comum em gatos do que em cães, devido à menor incidência de doença do disco intervertebral em gatos em comparação com cães (Lu et al., 2002; Chow et al., 2012). Maior consciência de Enpanc como diferencial para mielopatia aguda ou hiperaguda e não progressiva pode incentivar a utilização e a indicação de RM, pois essas ocorrências podem ter um bom resultado, como foi observado neste caso.

O tratamento realizado no paciente do presente relato foi conservativo, pois, como a principal etiologia da lesão medular secundária à Enpanc é a contusão associada a pouca ou nenhuma compressão, a descompressão cirúrgica, na maioria dos casos, não é indicada (Yarrow e Jeffery, 2000; Lu et al., 2002; Chang et al., 2007) .

A Enpanc é clinicamente semelhante à mielopatia isquêmica secundária à embolia fibrocartilaginosa (EPC), já que ambas são caracterizadas por início agudo e não progressivo. A etiopatogenia exata da mielopatia isquêmica por embolismo fibrocartilaginoso permanece desconhecida. $\mathrm{O}$ embolo de material fibrocartilaginoso foi demonstrado ser histologicamente idêntico ao material de núcleo pulposo. Teorias propostas para EPC como uma causa para a isquemia espinhal incluem entrada vascular do material do disco intervertebral via veias, artérias ou anastomoses arteriovenosas, entrada vascular do material do disco intervertebral via vascularização anômala no interior do disco (vasos embrionários remanescentes ou inflamação crônica) ou entrada do material do disco intervertebral em canais sinusoidais venosos vertebrais. Se EPC são, na verdade, fragmentos de núcleo pulposo não 
degenerados e o trauma é uma etiologia comum, então EPC e ENPANC podem ser manifestações de um mesmo processo da doença (Risio L e Platt, 2010. A presença de dor à palpação da coluna secundária à contusão e da inflamação das meninges é um dado clínico frequente na Enpanc e pôde ser observada no paciente deste relato. Em associação às imagens de RM, ela pode ajudar a diferenciar da EPC em que o processo isquêmico geralmente não leva à dor (Mckee et al., 2010).

Vários estudos de lesão medular traumática aguda em humanos identificaram uma associação entre a gravidade dos sinais neurológicos detectados na avaliação inicial, o grau de lesão da medula espinhal visualizado por meio de ressonância magnética e o resultado (MiyangI et al., 2007). Uma associação entre o resultado e a gravidade dos sinais neurológicos (perda de nocicepção) e a extensão da lesão nas imagens de RM (área de hiperintensidade intramedular nas imagens sagitais ponderadas em T2 superior ou igual ao comprimento do corpo vertebral de L2) foi detectada em um estudo com 77 cães paraplégicos com extrusão e degeneração do disco intervertebral Hansen tipo I (Ito et al., 2005). No presente relato, o paciente apresentou uma recuperação neurológica satisfatória, apesar da gravidade e da extensão da lesão na medula espinhal nas imagens de RM. Estudos prospectivos maiores são necessários para se obterem mais informações sobre as mudanças dos achados da RM com a evolução do caso e sua correlação com o tempo de recuperação, o prognóstico e o resultado em cães com Enpanc.

\section{CONCLUSÃO}

Conclui-se que, embora um diagnóstico definitivo de Enpanc só seja possível post mortem, os resultados deste relato sugerem que a RM pode ser útil para fazer um diagnóstico presuntivo em cães com histórico e sinais clínicos compatíveis. Pode também ajudar a diferenciar a Enpanc da FCE, que possuem um quadro clínico similar, e ajudar novos estudos de prognóstico e evolução. Além disso, o tratamento conservativo em extrusões discais não compressivas é preconizado e, apesar de uma grande extensão da lesão medular ser uma fator de prognóstico ruim, o paciente pode apresentar boa recuperação se a sensibilidade profunda não for perdida, como foi observado neste caso.

\section{REFERÊNCIAS}

CHANG, Y.; DENNIS, R.; PLATT, S.R.; PENDERIS, J. Magnetic resonance imaging of traumatic intervertebral disc extension in dogs. Vet. Rec., v.160, p.795-799, 2007.

CHOW, K.; BEATTY, J.A.; VOSS, K.; BARRS, V.R. Probable lumbar acute non-compressive nucleus pulposus extrusion in a cat with acute onset paraparesis. J. Feline Med. Surg., v.4, p.764-767, 2012.

GRIFFITHS, I. A syndrome produced by dorso-lateral explosions of the cervical intervertebral discs. Vet. Rec., v.87, p737-741, 1970.

HENKE, D.; GORGAS, D.; FLEGEL, T. et al. Magnetic resonance imaging findings in dogs with traumatic intervertebral disk extrusion with or without spinal cord compression: 31 cases (2006-2010). J. Am. Vet. Med. Assoc., v.242, p.217-222, 2013.

ITO, D.; MATSUNAGA, S.; JEFFERY, N.D. Prognostic value of magnetic resonance imaging in dogs with paraplegia caused by thoracolumbar intervertebral disk extrusion: 77 case (2000-2003). J. Am. Vet. Med. Assoc., v.227, p.1454-1460, 2005.

LU, D.; LAMB， C.R.; WESSELINGH， K.; TARGETT, M.P. Acute intervertebral disc extrusion in a cat: clinical and MRI findings J. Feline Med. Surg., v.4, p.65-68, 2002.

MCKEE, W.M.; DOWNES, C.J.; PINK, J.J.; GEMMILL, T.J. Presumptive exercise-associated peracute thoracolumbar disc extrusion in 48 dogs. Vet. Rec., v.166, p.523-528, 2010.

MIYANJI, F.; FURLAN, J.C.; AARABI, B. Acute cervical traumatic spinal cord injury: MR imaging findings correlated with neurologic outcomeprospective study with 100 consecutive patients. Radiology, v.243, p.820-827, 2007.

RISIO, L.; ADAMS, V.; DENNIS, R.; MCCONNELL, F.J. Association of clinical and magnetic resonance imaging findings with outcome in dogs with presumptive acute non-compressive nucleus pulposus extrusion: 42 cases (2000-2007). J. Am. Vet. Med. Assoc., v.234, p.495-504, 2009.

RISIO, L.; PLATT, S.R. Fibrocartilagenous embolic myelopathy in small animals. Vet. Clin. N. Am. Small Anim. Pract., v.40, p.859-869, 2010.

YARROW, T.G.; JEFFERY, N.D. Dura mater laceration associated with acute paraplegia in three dogs. Vet. Rec., v.146, p.138-139, 2000. 